\title{
Review Article \\ Role of Kallistatin Treatment in Aging and Cancer by Modulating miR-34a and miR-21 Expression
}

\author{
Julie Chao, Youming Guo, Pengfei Li, and Lee Chao \\ Department of Biochemistry and Molecular Biology, Medical University of South Carolina, Charleston, SC, USA \\ Correspondence should be addressed to Julie Chao; chaoj@musc.edu
}

Received 6 April 2017; Accepted 17 May 2017; Published 28 June 2017

Academic Editor: Jaideep Banerjee

Copyright (C) 2017 Julie Chao et al. This is an open access article distributed under the Creative Commons Attribution License, which permits unrestricted use, distribution, and reproduction in any medium, provided the original work is properly cited.

\begin{abstract}
Kallistatin is an endogenous protein that regulates differential signaling pathways and a wide spectrum of biological activities via its two structural elements: an active site and a heparin-binding domain. Kallistatin via its heparin-binding site inhibits vascular inflammation and oxidative stress by antagonizing TNF- $\alpha$-induced NADPH oxidase activity, NF- $\kappa \mathrm{B}$ activation, and inflammatory gene expression in endothelial cells. Moreover, kallistatin via its active site inhibits microRNA-34a (miR-34a) synthesis and stimulates eNOS and SIRT1 expression in endothelial progenitor cells, whereas its heparin-binding site is crucial for blocking TNF- $\alpha$-induced miR-21 expression and oxidative stress, thus reducing cellular senescence. By downregulating miR-34a and miR-21 expression, kallistatin treatment attenuates oxidative damage and aortic senescence in streptozotocin-induced diabetic mice and extends Caenorhabditis elegans lifespan under stress conditions. Likewise, kallistatin through the heparin-binding site inhibits TGF- $\beta$-induced miR-21 synthesis and oxidative stress in endothelial cells, resulting in inhibition of endothelial-mesenchymal transition, a process contributing to fibrosis and cancer. Furthermore, kallistatin's active site is essential for stimulating miR-34a and p53 expression and inhibiting the miR-21-Akt-Bcl-2 signaling pathway, thus inducing apoptosis in breast cancer cells. These findings reveal novel mechanisms of kallistatin in protection against senescence, aging, and cancer development by modulating miR-34a and miR-21 levels and inhibiting oxidative stress.
\end{abstract}

\section{Introduction}

Aging, characterized by the deterioration of human physiological functions, is the dominant risk factor for the development of cardiovascular disease and cancer [1]. Aging and cancer share common origins, as increased production of reactive oxygen species (ROS) plays a critical role in oxidative damage in aged cells and tumor progression $[2,3]$. Thus, identifying new antioxidant agents to delay aging and reduce cancer development is imperative in pharmacological intervention. Moreover, microRNAs (miRNAs) negatively regulate the expression of target genes at the posttranscriptional level and play a pivotal role in cellular senescence and tumor progression $[4,5]$. Among miRNAs, miR-21 has a widely described oncogenic function by targeting multiple signaling pathways in regulating cancer cell apoptosis, migration, invasion, proliferation, and angiogenesis [6,7]. Conversely, miR-34a inhibits cancer cell survival, proliferation, invasion, and metastasis formation $[8,9]$. Thus, miR-21 functions as an oncogene, while miR-34a acts as a tumor suppressor. However, both miR-34a and miR-21 promote cellular senescence and are crucial players in the regulation of aging and cancer development.

miR-21 promotes tumor progression by stimulating ROS production through downregulation of superoxide dismutase (SOD2/SOD3) [10]. Besides its association with oxidative stress, miR-21 has a potential role in fibrosis, as inhibition of miR-21 attenuates fibrosis in the heart, kidney, and lungs [11-13]. Transforming growth factor- (TGF-) $\beta 1$, via stimulation of ROS formation, induces upregulation of miR-21, leading to organ fibrosis [14]. Moreover, miR-21 plays an important role in TGF- $\beta$-induced endothelial-tomesenchymal transition (EndMT), a process that contributes to carcinoma-associated fibrosis and fibrotic disease [15]. Therefore, miR-21 exhibits a functional interplay with oxidative stress during tumorigenesis and fibrosis. Furthermore, miR-21 promotes cellular senescence by suppressing high-mobility group A2 in endothelial progenitor cells 
(EPCs) [16]. Conversely, miR-34a exerts controversial functions in cancer development and the aging process, as it functions as a tumor suppressor and a senescence inducer $[17,18]$. miR-34a inhibits cancer progression by inhibiting cancer cell survival, proliferation, invasion, and metastasis formation $[8,9]$, while it promotes renal cell senescence by inhibiting antioxidant enzymes [19]. miR-34a induces cellular senescence through suppression of the longevity gene sirtuin 1 (SIRT1) in endothelial cells [18]. Overexpression of miR-34a induces premature senescence of young mesangial cells via downregulation of SOD2, leading to an increase in ROS generation, while antisense miR-34a inhibits senescence of old mesangial cells via upregulation of SOD2 and a decrease in ROS [19]. Collectively, these findings indicate that miR-21 and miR-34a are pivotal players in aging and tumor progression.

Kallistatin was first identified in human plasma as a tissue kallikrein inhibitor and a serine proteinase inhibitor [20-23]. Kallistatin is widely distributed in tissues relevant to cardiovascular function, including those of the kidney, heart, and blood vessels [24-26]. Kallistatin contains two structural elements: an active site and a heparin-binding domain [27-29]. The active site of kallistatin is a key for inhibiting tissue kallikrein activity and stimulating endothelial nitric oxide synthase (eNOS) and SIRT1 expression [30, 31]. Kallistatin via its heparin-binding site interacts with cell surface heparan sulfate proteoglycans, thus antagonizing signaling pathways mediated by vascular endothelial growth factor (VEGF), tumor necrosis factor- (TNF-) $\alpha$, high-mobility group box1 (HMGB1), TGF- $\beta$, and epidermal growth factor (EGF) [31-35]. Kallistatin administration exhibits pleiotropic effects, including reduction in blood pressure and inhibition of oxidative stress, inflammation, angiogenesis, apoptosis, hypertrophy, and fibrosis in animal models [33, 36-42]. Kallistatin inhibits TNF- $\alpha$-induced ROS production, nuclear factor- (NF-) $\kappa \mathrm{B}$ activation, and inflammatory gene expression in endothelial cells $[33,42]$. In addition, kallistatin reduces TNF- $\alpha$-induced endothelial senescence and delays stress-induced aging by inhibiting miR-34a, miR-21, and oxidative stress and upregulating the expression of the antioxidant enzymes eNOS, SIRT1, catalase, and SOD3 in cultured EPCs, streptozotocin- (STZ-) induced diabetic mice, and Caenorhabditis elegans (C. elegans). Moreover, kallistatin treatment prevents TGF- $\beta$-induced EndMT by reducing ROS formation and miR-21 synthesis [31]. Furthermore, kallistatin induces apoptosis by stimulating miR-34a and suppressing miR-21 synthesis in breast cancer cells [43]. This review focuses on the protective role of kallistatin in cellular senescence, aging, and tumor progression by inhibiting oxidative stress and regulating miR-34a and miR-21 levels.

\section{Kallistatin Inhibits Oxidative Stress and Inflammation}

Oxidative stress stimulates inflammatory pathways, which promotes cellular senescence and aging [44]. Besides superoxide radical $\left(\mathrm{O}_{2}^{-}\right), \mathrm{NF}-\kappa \mathrm{B}$ activation also links to accelerated aging in systemic inflammatory responses [45]. Oxidative stress and activation of $\mathrm{NF}-\kappa \mathrm{B}$ have been shown to be associated with senescence of cultured endothelial cells, and pharmacological inhibition of NF- $\kappa \mathrm{B}$ signaling prevents age-associated features in mouse models [46]. Reduced kallistatin levels are correlated with increased oxidative stress, inflammation, and organ damage in animal models of hypertension, cardiovascular, and renal damage [22, 35, 40, 42]. Kallistatin treatment reduces ROS generation, inflammation, and organ damage associated with increased eNOS and NO levels in several animal models, including acute and chronic myocardial damage, salt-induced hypertension, and sepsis $[34,39,40,42]$. On the other hand, depletion of endogenous kallistatin by neutralizing antibody injection further increases oxidative stress, inflammation, and fibrosis and augments cardiovascular and renal damage in salt-induced hypertensive rats [47]. These findings indicate that kallistatin has a protective role in cardiovascular and renal dysfunction by inhibition of oxidative stress and inflammation.

Kallistatin is an antioxidant as its heparin-binding site is essential for blocking TNF- $\alpha$-induced NADPH oxidase activity and expression in endothelial cells $[33,42]$. In addition, kallistatin's active site is a key for stimulating the expression of the antioxidant enzymes eNOS, SIRT1, and catalase in endothelial cells and EPCs [31, 48]. Kallistatin inhibits $\mathrm{NAD}(\mathrm{P}) \mathrm{H}$ oxidase activity and ROS formation, partly by NO formation in cardiomyocytes [39]. Kallistatin attenuates vascular inflammation by antagonizing TNF- $\alpha$ and HMGB1-mediated NF- $\kappa \mathrm{B}$ activation and expression of proinflammatory genes in endothelial cells [33, 34]. Moreover, kallistatin stimulates eNOS expression by interacting with the transcription factor Kruppel-like factor 4 and increases eNOS activity and NO generation by activating the phosphoinositide 3-kinase- (PI3K-) Akt signaling pathway [41]. Kallistatin not only stimulates eNOS expression but also prevents TNF- $\alpha$-mediated inhibition of eNOS synthesis in endothelial cells [31]. NO production can inhibit inflammatory gene expression by preventing activation of $\mathrm{NF}-\kappa \mathrm{B}[49]$. These findings indicate that kallistatin, through its antioxidative and anti-inflammatory effects, protects against multiorgan damage and accelerated aging.

\section{Kallistatin Reduces Vascular Senescence and Aging by Downregulating miR-34a and miR-21 Synthesis}

miR-34a and miR-21 have emerged as important regulators of cellular senescence and aging [50]. miR-34a functions as a main senescence promoter by inhibiting the expression of SIRT1, a conservative longevity gene, through a miR-34abinding site within the $3^{\prime}$-UTR of SIRT1 [51]. Likewise, miR-21 is involved in accelerating cellular senescence in EPCs [16]. Moreover, oxidative stress induces vascular injury and endothelial senescence, with the inflammatory cytokine TNF- $\alpha$ being the main contributor to ROS production [52]. SIRT1 accounts for vascular homeostasis by activating many antioxidant enzymes, such as eNOS, catalase, and manganese superoxide dismutase (MnSOD), to diminish ROS [53]. Upregulation of antioxidant proteins, such as eNOS, SIRT1, catalase, and MnSOD, protects against oxidative stress- 


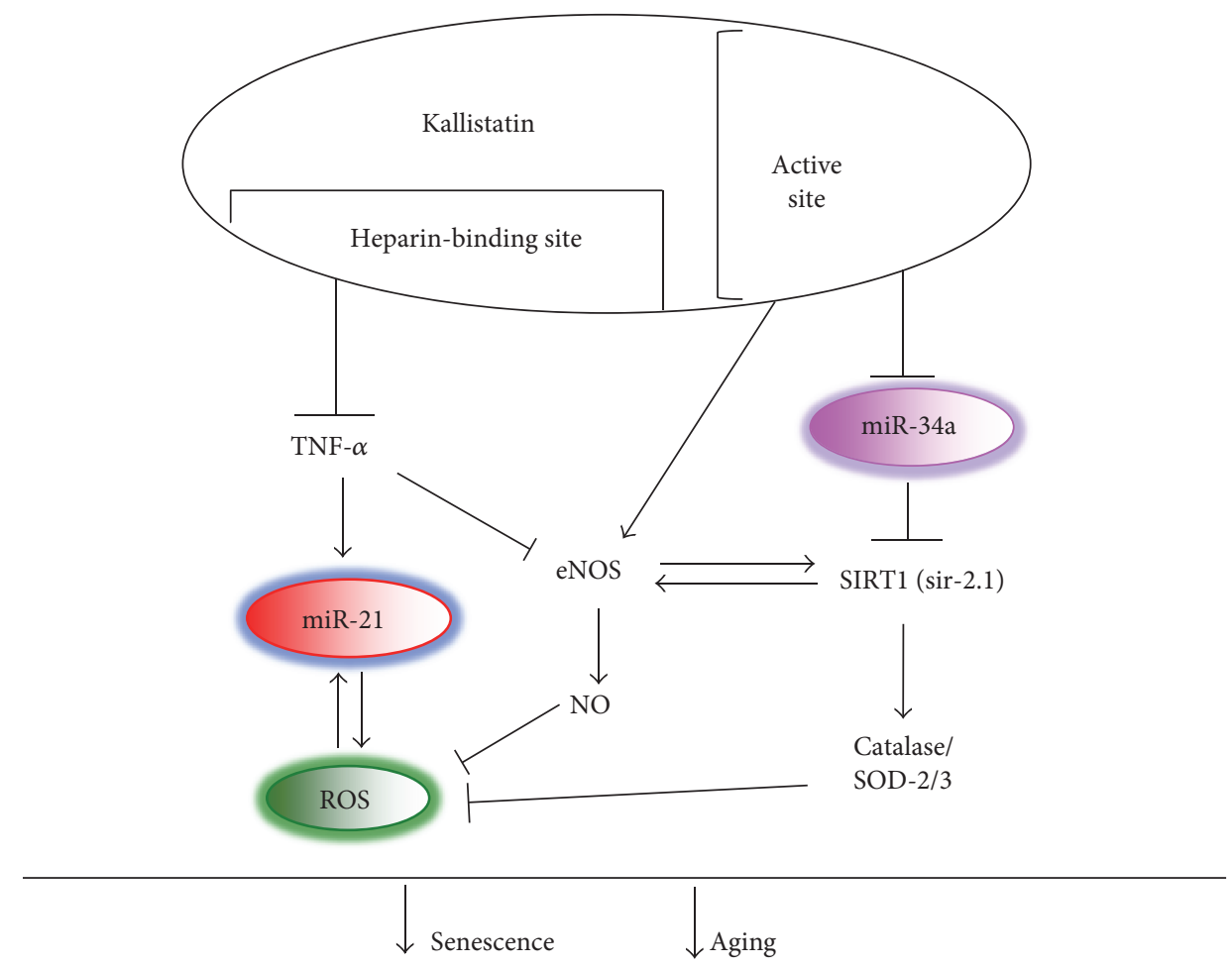

FIgURE 1: Signaling mechanism by which kallistatin inhibits senescence and aging through suppressing miR-34a expression and TNF- $\alpha$-induced miR-21-ROS production and stimulating eNOS/SIRT1-NO levels in EPCs.

mediated insults $[54,55]$. Therefore, prosenescent miR-34a and miR-21, as well as antioxidant enzymes, underlie the molecular basis for endothelial senescence and agingassociated diseases.

Kallistatin treatment significantly reduces TNF- $\alpha$ induced senescence in EPCs, as indicated by reduced senescence-associated $\beta$-galactosidase activity and elevated telomerase activity. Telomerase prevents telomere attrition by synthesis of telomeric repeats onto the $3^{\prime}$ end of telomeres. Telomere shortening is observed during normal aging in humans and mice [56]. Circulatory kallistatin levels are positively associated with leucocyte telomere length in humans [57], indicating a potential role of kallistatin in maintaining telomere length and attenuating the aging process. In addition, kallistatin via its heparin-binding site antagonizes TNF- $\alpha$-induced superoxide levels and miR-21 synthesis, as well as TNF- $\alpha$-mediated inhibition of SIRT1, eNOS, and catalase synthesis in EPCs. Kallistatin via its active site inhibits miR-34a synthesis but stimulates the expression of antioxidant enzymes in EPCs. Overexpression of miR-34a abolishes kallistatin's effects on SIRT1 and eNOS and its antisenescence activity. Kallistatin administration attenuates aortic senescence, oxidative stress, and miR-34a and miR-21 synthesis, in association with elevated SIRT1, eNOS, and catalase levels in STZ-induced diabetic mice. Furthermore, human kallistatin treatment prolongs the lifespan of wild-type C. elegans under heat or oxidative stress conditions but has no effect on miR-34 or sir-2.1 (SIRT1 homolog) C. elegans mutants. Similar to diabetic mice, kallistatin inhibits miR34 and superoxide formation but stimulates sir-2.1 synthesis in C. elegans. The signaling mechanism of kallistatin in senescence and aging by regulating miR-34a and miR-21 levels is shown in Figure 1. These combined findings provide significant insights into the novel role and mechanism of kallistatin in endothelial senescence and aging.

\section{Kallistatin Inhibits EndMT via Suppressing TGF- $\beta$-Induced Oxidative Stress and miR-21 Expression}

Endothelial-mesenchymal transition (EndMT) contributes greatly to organ fibrosis and tumor metastasis $[58,59]$. Morphological changes in EndMT induced by TGF- $\beta$ signaling cascades include loss of cell-cell junctions and endothelial markers, such as vascular endothelial- (VE-) cadherin and $\mathrm{CD} 31$, and gain of the mesenchymal marker $\alpha$-smooth muscle actin (SMA). Moreover, miR-21 expression levels are highly elevated during EMT and EndMT $[15,60]$. TGF- $\beta$ induced EndMT is partly mediated by upregulation of the miR-21-Akt pathway, as blockade of miR-21 can prevent EndMT and inhibit Akt activation [15]. Furthermore, ROS production further stimulates miR-21 synthesis in fibroblasts [61]. Therefore, miR-21 is a key mediator of EndMT, as well as EndMT-associated fibrosis and tumor development.

Human kallistatin delivery exerts beneficial effects on fibrosis by suppressing TGF- $\beta$ synthesis in animal models $[40,62,63]$. Kallistatin treatment blocks TGF- $\beta$-induced EndMT in endothelial cells, as evidenced by morphological changes, increased endothelial markers (VE-cadherin and CD31), and reduced mesenchymal marker ( $\alpha$-SMA) [31]. Kallistatin prevents TGF- $\beta$-mediated activation of the miR- 


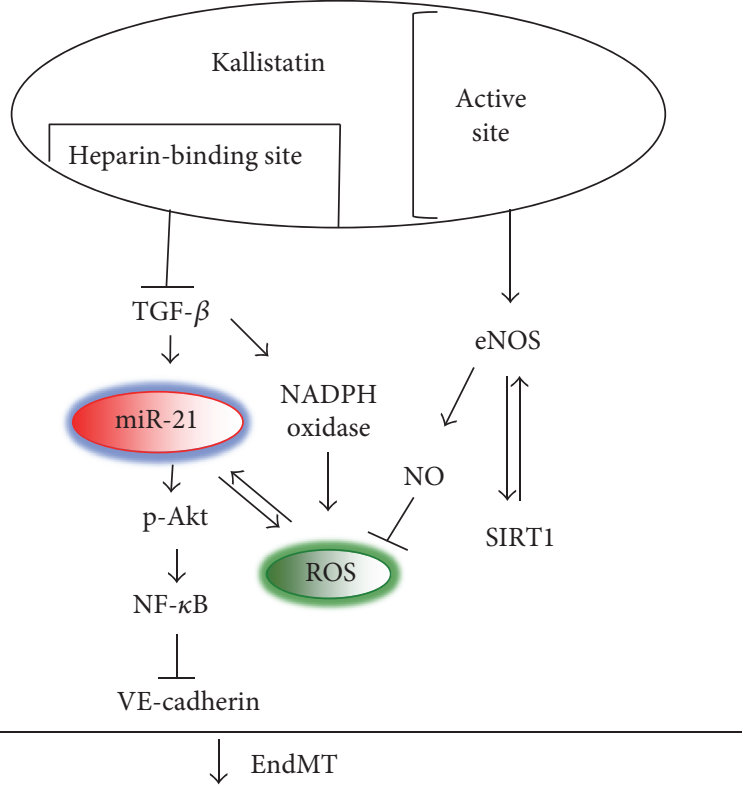

FIgURE 2: Signaling mechanism by which kallistatin inhibits EndMT by preventing the TGF- $\beta$-induced miR-21-Akt-NF- $\kappa \mathrm{B}$ pathway and oxidative stress and stimulating eNOS/SIRT1 expression in endothelial cells.

21-phospho-Akt-NF- $\kappa \mathrm{B}$ signaling pathway, as well as TGF$\beta$-induced NADPH oxidase expression and activity, and ROS formation [31]. Kallistatin's heparin-binding site is crucial for inhibiting TGF- $\beta$-induced oxidative stress, while its active site is a key for stimulating expression of the antioxidant proteins eNOS and SIRT1 and production of NO. Moreover, kallistatin via the heparin-binding site blocks TGF- $\beta$-induced miR-2 1 expression, Akt phosphorylation, and NF- $\kappa \mathrm{B}$ activation. Thus, kallistatin inhibits EndMT through suppressing the TGF- $\beta$-induced miR-21-Akt-NF$\kappa \mathrm{B}$ signaling pathway and stimulating antioxidant protein expression (Figure 2). These findings indicate that kallistatin attenuates fibrosis and cancer by suppressing TGF- $\beta$ induced EndMT.

\section{Kallistatin Induces Cancer Cell Apoptosis by Stimulating miR-34a and Inhibiting miR-21 Expression}

miRNAs are well categorized by their cancer-related functions, including tumor suppression, oncogene expression, epithelial-mesenchymal transition, apoptosis, and immune response [64]. miR-34a plays an important role as a tumor suppressor in many types of cancers [65]. Indeed, miR-34a levels are underexpressed in a variety of human tumors, and low levels of miR-34 have been related to poor clinical outcome of cancer patients $[66,67]$. Moreover, miR-34a inhibits the proliferation, invasion, and migration of breast cancer cells and breast tumor growth in vivo by deactivating the Wnt/ $\beta$-catenin signaling pathway [68]. On the other hand, miR-21, a well-recognized tumor inducer, is upregulated in a large range of human tumors, including gastric,

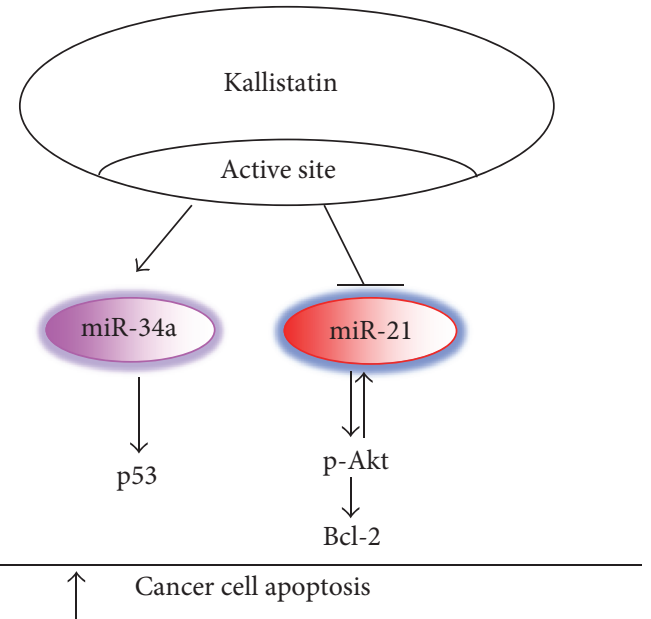

FIGURE 3: Signaling mechanism by which kallistatin induces apoptosis through upregulating miR-34a-p53 and downregulating miR-21-Akt-Bcl-2 pathways in breast cancer cells.

colorectal, lung, pancreatic, ovarian, and breast cancer [6974]. In addition, high levels of miR-21 expression are strongly related to poor clinical prognosis of patients in pancreatic cancer [75]. The prosurvival protein $\mathrm{Bcl}-2$, a key regulator of apoptosis in many types of human tumors, is positively regulated by miR-21, and an anti-miR-21 inhibitor downregulates Bcl-2 in breast cancer cells [76]. Moreover, resveratrol induces bladder cancer cell apoptosis by reducing miR-21 expression, Akt phosphorylation, and Bcl-2 levels [77]. Therefore, these findings indicate opposite effects of miR$34 \mathrm{a}$ and miR-21 in cancer development.

Kallistatin gene transfer has been reported to inhibit tumor growth and metastasis in several animal models [32, 78-82]. Local administration of human kallistatin reduces tumor growth and angiogenesis in nude mice via antagonizing VEGF-mediated proliferation, migration, and invasion of cultured endothelial cells [32, 78]. Moreover, kallistatin induces apoptotic cell death in human colorectal cancer cells [83]. Kallistatin via the active site stimulates miR-34a and suppresses miR-21 expression in breast cancer cells [44]. Kallistatin reduces cancer cell viability and induces apoptosis by increasing miR-34a and $\mathrm{p} 53$ expression but reducing miR21 synthesis, Akt phosphorylation, and $\mathrm{Bcl}-2$ expression in breast cancer cells [44]. Thus, kallistatin induces breast cancer cell apoptosis by stimulating miR-34a-p53 and suppressing miR-21-Akt-Bcl-2 signaling pathways (Figure 3 ). These findings indicate that kallistatin induces cancer cell death through upregulation of $\mathrm{miR}-34 \mathrm{a}$ and downregulation of miR-21 expression.

\section{Conclusion}

Kallistatin plays a protective role in accelerated aging and cancer development by regulation of miR-34a and miR-21. Kallistatin's antisenescence/aging effect is mainly attributed to suppression of oxidative stress and inflammation and downregulation of miR-34a and miR-21 synthesis. Kallistatin via its heparin-binding domain antagonizes TNF- $\alpha$-induced ROS formation and miR-21 expression, while its active site 
is crucial for inhibiting miR-34a synthesis in EPCs. Kallistatin protects against fibrosis and tumorigenesis by inhibiting EndMT. Kallistatin's heparin-binding site is critical for suppressing TGF- $\beta$-induced ROS formation and the miR-21Akt-NF- $\kappa$ B signaling pathway in endothelial cells. However, kallistatin via its active site stimulates miR-34a and p53 synthesis and inhibits miR-21-Akt-Bcl-2 signaling, leading to apoptosis in breast cancer cells. As an endogenous protein, kallistatin treatment could improve human health during aging process and tumor progression with minimal side effects. This review article provides a new potential prospective in kallistatin-based therapeutic intervention in aging and cancer.

\section{Conflicts of Interest}

The authors declare that there is no conflict of interests regarding the publication of this paper.

\section{Authors' Contributions}

Julie Chao contributes to the writing, and Youming Guo, Pengfei $\mathrm{Li}$, and Lee Chao contribute to the revision and citation of the paper.

\section{Acknowledgments}

This work was supported by the National Institutes of Health grant (HL118516).

\section{References}

[1] C. Lopez-Otin, M. A. Blasco, L. Partridge, M. Serrano, and G. Kroemer, "The hallmarks of aging," Cell, vol. 153, no. 6, pp. 1194-1217, 2013.

[2] P. Storz, "Reactive oxygen species in tumor progression," Frontiers in Bioscience, vol. 10, pp. 1881-1896, 2005.

[3] P. Davalli, T. Mitic, A. Caporali, A. Lauriola, and D. D'Arca, "ROS, cell senescence, and novel molecular mechanisms in aging and age-related diseases," Oxidative Medicine and Cellular Longevity, vol. 2016, Article ID 3565127, 18 pages, 2016.

[4] D. P. Bartel, "MicroRNAs: genomics, biogenesis, mechanism, and function," Cell, vol. 116, no. 2, pp. 281-297, 2004.

[5] F. Olivieri, M. R. Rippo, V. Monsurro et al., "MicroRNAs linking inflamm-aging, cellular senescence and cancer," Ageing Research Reviews, vol. 12, no. 4, pp. 1056-1068, 2013.

[6] B. Zhang, X. Pan, G. P. Cobb, and T. A. Anderson, "microRNAs as oncogenes and tumor suppressors," Developmental Biology, vol. 302, no. 1, pp. 1-12, 2007.

[7] P. Olson, J. Lu, H. Zhang et al., "MicroRNA dynamics in the stages of tumorigenesis correlate with hallmark capabilities of cancer," Genes \& Development, vol. 23, no. 18, pp. 2152-2165, 2009.

[8] Y. Guo, S. Li, J. Qu et al., "MiR-34a inhibits lymphatic metastasis potential of mouse hepatoma cells," Molecular and Cellular Biochemistry, vol. 354, no. 1-2, pp. 275-282, 2011.

[9] L. Sun, Z. Wu, Y. Shao et al., "MicroRNA-34a suppresses cell proliferation and induces apoptosis in U87 glioma stem cells," Technology in Cancer Research \& Treatment, vol. 11, no. 5, pp. 483-490, 2012.
[10] X. Zhang, W. L. Ng, P. Wang et al., "MicroRNA-21 modulates the levels of reactive oxygen species by targeting SOD3 and TNFalpha," Cancer Research, vol. 72, no. 18, pp. 4707-4713, 2012.

[11] G. Liu, A. Friggeri, Y. Yang et al., "miR-21 mediates fibrogenic activation of pulmonary fibroblasts and lung fibrosis," The Journal of Experimental Medicine, vol. 207, no. 8, pp. 15891597, 2010.

[12] T. Thum, N. Chau, B. Bhat et al., "Comparison of different miR-21 inhibitor chemistries in a cardiac disease model," The Journal of Clinical Investigation, vol. 121, no. 2, pp. 461462, 2011, author reply 462-463.

[13] X. Zhong, A. C. Chung, H. Y. Chen, X. M. Meng, and H. Y. Lan, "Smad3-mediated upregulation of miR-21 promotes renal fibrosis," Journal of the American Society of Nephrology, vol. 22, no. 9, pp. 1668-1681, 2011.

[14] M. Fierro-Fernandez, V. Miguel, and S. Lamas, "Role of redoximiRs in fibrogenesis," Redox Biology, vol. 7, pp. 58-67, 2016.

[15] R. Kumarswamy, I. Volkmann, V. Jazbutyte, S. Dangwal, D. H. Park, and T. Thum, "Transforming growth factor-betainduced endothelial-to-mesenchymal transition is partly mediated by microRNA-21," Arteriosclerosis, Thrombosis, and Vascular Biology, vol. 32, no. 2, pp. 361-369, 2012.

[16] S. Zhu, S. Deng, Q. Ma et al., "MicroRNA-10A* and microRNA-21 modulate endothelial progenitor cell senescence via suppressing high-mobility group A2," Circulation Research, vol. 112, no. 1, pp. 152-164, 2013.

[17] H. Hermeking, "The miR-34 family in cancer and apoptosis," Cell Death and Differentiation, vol. 17, no. 2, pp. 193-199, 2010.

[18] T. Ito, S. Yagi, and M. Yamakuchi, "MicroRNA-34a regulation of endothelial senescence," Biochemical and Biophysical Research Communications, vol. 398, no. 4, pp. 735-740, 2010.

[19] X. Y. Bai, Y. Ma, R. Ding, B. Fu, S. Shi, and X. M. Chen, "miR-335 and miR-34a promote renal senescence by suppressing mitochondrial antioxidative enzymes," Journal of the American Society of Nephrology, vol. 22, no. 7, pp. 1252-1261, 2011.

[20] J. Chao, D. M. Tillman, M. Y. Wang, H. S. Margolius, and L. Chao, "Identification of a new tissue-kallikrein-binding protein," The Biochemical Journal, vol. 239, no. 2, pp. 325-331, 1986.

[21] M. Y. Wang, J. Day, L. Chao, and J. Chao, "Human kallistatin, a new tissue kallikrein-binding protein: purification and characterization," Advances in Experimental Medicine and Biology, vol. 247B, pp. 1-8, 1989.

[22] J. Chao, K. X. Chai, L. M. Chen et al., "Tissue kallikreinbinding protein is a serpin. I. Purification, characterization, and distribution in normotensive and spontaneously hypertensive rats," The Journal of Biological Chemistry, vol. 265, no. 27, pp. 16394-16401, 1990.

[23] G. X. Zhou, L. Chao, and J. Chao, "Kallistatin: a novel human tissue kallikrein inhibitor. Purification, characterization, and reactive center sequence," The Journal of Biological Chemistry, vol. 267, no. 36, pp. 25873-25880, 1992.

[24] J. Chao and L. Chao, "Biochemistry, regulation and potential function of kallistatin," Biological Chemistry Hoppe-Seyler, vol. 376, no. 12, pp. 705-713, 1995.

[25] L. M. Chen, Q. Song, L. Chao, and J. Chao, "Cellular localization of tissue kallikrein and kallistatin mRNAs in human kidney," Kidney International, vol. 48, no. 3, pp. 690-697, 1995. 
[26] W. C. Wolf, R. A. Harley, D. Sluce, L. Chao, and J. Chao, "Localization and expression of tissue kallikrein and kallistatin in human blood vessels," The Journal of Histochemistry and Cytochemistry, vol. 47, no. 2, pp. 221-228, 1999.

[27] V. C. Chen, L. Chao, and J. Chao, "Reactive-site specificity of human kallistatin toward tissue kallikrein probed by site-directed mutagenesis," Biochimica et Biophysica Acta, vol. 1479, no. 1-2, pp. 237-246, 2000.

[28] V. C. Chen, L. Chao, and J. Chao, "Roles of the P1, P2, and P3 residues in determining inhibitory specificity of kallistatin toward human tissue kallikrein," The Journal of Biological Chemistry, vol. 275, no. 49, pp. 38457-38466, 2000.

[29] V. C. Chen, L. Chao, D. C. Pimenta, G. Bledsoe, L. Juliano, and J. Chao, "Identification of a major heparin-binding site in kallistatin," The Journal of Biological Chemistry, vol. 276, no. 2, pp. 1276-1284, 2001.

[30] V. C. Chen, L. Chao, and J. Chao, "A positively charged loop on the surface of kallistatin functions to enhance tissue kallikrein inhibition by acting as a secondary binding site for kallikrein," The Journal of Biological Chemistry, vol. 275, no. 51, pp. 40371-40377, 2000.

[31] Y. Guo, P. Li, G. Bledsoe, Z. R. Yang, L. Chao, and J. Chao, "Kallistatin inhibits TGF-beta-induced endothelialmesenchymal transition by differential regulation of microRNA-21 and eNOS expression," Experimental Cell Research, vol. 337, no. 1, pp. 103-110, 2015.

[32] R. Q. Miao, V. Chen, L. Chao, and J. Chao, "Structural elements of kallistatin required for inhibition of angiogenesis," American Journal of Physiology Cell Physiology, vol. 284, no. 6, pp. C1604-C1613, 2003.

[33] H. Yin, L. Gao, B. Shen, L. Chao, and J. Chao, "Kallistatin inhibits vascular inflammation by antagonizing tumor necrosis factor-alpha-induced nuclear factor kappaB activation," Hypertension, vol. 56, no. 2, pp. 260-267, 2010.

[34] P. Li, G. Bledsoe, Z. R. Yang, H. Fan, L. Chao, and J. Chao, "Human kallistatin administration reduces organ injury and improves survival in a mouse model of polymicrobial sepsis," Immunology, vol. 142, no. 2, pp. 216-226, 2014.

[35] J. Chao, G. Bledsoe, and L. Chao, "Protective role of kallistatin in vascular and organ injury," Hypertension, vol. 68, no. 3, pp. 533-541, 2016.

[36] L. M. Chen, L. Chao, and J. Chao, “Adenovirus-mediated delivery of human kallistatin gene reduces blood pressure of spontaneously hypertensive rats," Human Gene Therapy, vol. 8, no. 3, pp. 341-347, 1997.

[37] C. R. Wang, S. Y. Chen, C. L. Wu et al., "Prophylactic adenovirus-mediated human kallistatin gene therapy suppresses rat arthritis by inhibiting angiogenesis and inflammation," Arthritis and Rheumatism, vol. 52, no. 4, pp. 1319-1324, 2005.

[38] J. Chao, H. Yin, Y. Y. Yao, B. Shen, R. S. Smith Jr., and L. Chao, "Novel role of kallistatin in protection against myocardial ischemia-reperfusion injury by preventing apoptosis and inflammation," Human Gene Therapy, vol. 17, no. 12, pp. 1201-1213, 2006.

[39] L. Gao, H. Yin, S. R. Smith Jr., L. Chao, and J. Chao, "Role of kallistatin in prevention of cardiac remodeling after chronic myocardial infarction," Laboratory Investigation, vol. 88, no. 11, pp. 1157-1166, 2008.

[40] B. Shen, M. Hagiwara, Y. Y. Yao, L. Chao, and J. Chao, "Salutary effect of kallistatin in salt-induced renal injury, inflammation, and fibrosis via antioxidative stress," Hypertension, vol. 51, no. 5, pp. 1358-1365, 2008.

[41] B. Shen, R. S. Smith Jr., Y. T. Hsu, L. Chao, and J. Chao, "Kruppel-like factor 4 is a novel mediator of kallistatin in inhibiting endothelial inflammation via increased endothelial nitric-oxide synthase expression," The Journal of Biological Chemistry, vol. 284, no. 51, pp. 35471-35478, 2009.

[42] B. Shen, L. Gao, Y. T. Hsu et al., "Kallistatin attenuates endothelial apoptosis through inhibition of oxidative stress and activation of Akt-eNOS signaling," American Journal of Physiology Heart and Circulatory Physiology, vol. 299, no. 5, pp. H1419-H1427, 2010.

[43] P. Li, Y. Guo, G. Bledsoe, Z. Yang, L. Chao, and J. Chao, "Kallistatin induces breast cancer cell apoptosis and autophagy by modulating Wnt signaling and microRNA synthesis," Experimental Cell Research, vol. 340, no. 2, pp. 305-314, 2016.

[44] J. Wu, S. Xia, B. Kalionis, W. Wan, and T. Sun, "The role of oxidative stress and inflammation in cardiovascular aging," BioMed Research International, vol. 2014, Article ID 615312, 13 pages, 2014.

[45] C. Soria-Valles, F. G. Osorio, A. Gutierrez-Fernandez et al., "NF-kappaB activation impairs somatic cell reprogramming in ageing," Nature Cell Biology, vol. 17, no. 8, pp. 1004-1013, 2015.

[46] S. Y. Khan, E. M. Awad, A. Oszwald et al., "Premature senescence of endothelial cells upon chronic exposure to TNFalpha can be prevented by $\mathrm{N}$-acetyl cysteine and plumericin," Scientific Reports, vol. 7, article 39501, 2017.

[47] Y. Liu, G. Bledsoe, M. Hagiwara, B. Shen, L. Chao, and J. Chao, "Depletion of endogenous kallistatin exacerbates renal and cardiovascular oxidative stress, inflammation, and organ remodeling," American Journal of Physiology Renal Physiology, vol. 303, no. 8, pp. F1230-F1238, 2012.

[48] L. Gao, P. Li, J. Zhang et al., "Novel role of kallistatin in vascular repair by promoting mobility, viability, and function of endothelial progenitor cells," Journal of the American Heart Association, vol. 3, no. 5, article e001194, 2014.

[49] J. R. Matthews, C. H. Botting, M. Panico, H. R. Morris, and R. T. Hay, "Inhibition of NF-kappaB DNA binding by nitric oxide," Nucleic Acids Research, vol. 24, no. 12, pp. 22362242, 1996.

[50] S. Dimmeler and P. Nicotera, "MicroRNAs in age-related diseases," EMBO Molecular Medicine, vol. 5, no. 2, pp. 180-190, 2013.

[51] T. Zhao, J. Li, and A. F. Chen, "MicroRNA-34a induces endothelial progenitor cell senescence and impedes its angiogenesis via suppressing silent information regulator 1," American Journal of Physiology Endocrinology and Metabolism, vol. 299, no. 1, pp. E110-E116, 2010.

[52] X. Chen, B. T. Andresen, M. Hill, J. Zhang, F. Booth, and C. Zhang, "Role of reactive oxygen species in tumor necrosis factor-alpha induced endothelial dysfunction," Current Hypertension Reviews, vol. 4, no. 4, pp. 245-255, 2008.

[53] M. Kitada, Y. Ogura, and D. Koya, "The protective role of Sirt1 in vascular tissue: its relationship to vascular aging and atherosclerosis," Aging, vol. 8, no. 10, pp. 2290-2307, 2016.

[54] S. Wassmann, K. Wassmann, and G. Nickenig, "Modulation of oxidant and antioxidant enzyme expression and function in vascular cells," Hypertension, vol. 44, no. 4, pp. 381-386, 2004.

[55] H. Ota, M. Eto, S. Ogawa, K. Iijima, M. Akishita, and Y. Ouchi, "SIRT1/eNOS axis as a potential target against vascular 
senescence, dysfunction and atherosclerosis," Journal of Atherosclerosis and Thrombosis, vol. 17, no. 5, pp. 431-435, 2010.

[56] M. A. Blasco, "Telomere length, stem cells and aging," Nature Chemical Biology, vol. 3, no. 10, pp. 640-649, 2007.

[57] H. Zhu, J. Chao, A. Raed et al., "Plasma kallistatin is associated with leukocyte telomere length in young African Americans," The FASEB Journal, vol. 30, no. 1, p. 625.1, 2016.

[58] E. M. Zeisberg, O. Tarnavski, M. Zeisberg et al., "Endothelialto-mesenchymal transition contributes to cardiac fibrosis," Nature Medicine, vol. 13, no. 8, pp. 952-961, 2007.

[59] S. Potenta, E. Zeisberg, and R. Kalluri, "The role of endothelialto-mesenchymal transition in cancer progression," British Journal of Cancer, vol. 99, no. 9, pp. 1375-1379, 2008.

[60] O. Bornachea, M. Santos, A. B. Martinez-Cruz et al., "EMT and induction of miR-21 mediate metastasis development in Trp53-deficient tumours," Scientific Reports, vol. 2, p. 434, 2012.

[61] M. Ling, Y. Li, Y. Xu et al., "Regulation of miRNA-21 by reactive oxygen species-activated ERK/NF-kappaB in arseniteinduced cell transformation," Free Radical Biology \& Medicine, vol. 52, no. 9, pp. 1508-1518, 2012.

[62] X. Huang, X. Wang, Y. Lv, L. Xu, J. Lin, and Y. Diao, "Protection effect of kallistatin on carbon tetrachloride-induced liver fibrosis in rats via antioxidative stress," PloS One, vol. 9, no. 2, article e88498, 2014.

[63] W. H. Yiu, D. W. Wong, H. J. Wu et al., "Kallistatin protects against diabetic nephropathy in $\mathrm{db} / \mathrm{db}$ mice by suppressing AGE-RAGE-induced oxidative stress," Kidney International, vol. 89, no. 2, pp. 386-398, 2016.

[64] C. Qiu, G. Chen, and Q. Cui, "Towards the understanding of microRNA and environmental factor interactions and their relationships to human diseases," Scientific Reports, vol. 2, p. 318, 2012.

[65] X. J. Li, Z. J. Ren, and J. H. Tang, "MicroRNA-34a: a potential therapeutic target in human cancer," Cell Death \& Disease, vol. 5, article e1327, 2014.

[66] D. Kong, E. Heath, W. Chen et al., "Epigenetic silencing of miR-34a in human prostate cancer cells and tumor tissue specimens can be reversed by BR-DIM treatment," American Journal of Translational Research, vol. 4, no. 1, pp. 14-23, 2012.

[67] S. Roy, E. Levi, A. P. Majumdar, and F. H. Sarkar, "Expression of miR-34 is lost in colon cancer which can be re-expressed by a novel agent CDF," Journal of Hematology \& Oncology, vol. 5, p. $58,2012$.

[68] W. Si, Y. Li, H. Shao et al., "miR-34a inhibits breast cancer proliferation and progression by targeting Wnt in Wnt/ beta-catenin signaling pathway," The American Journal of the Medical Sciences, vol. 352, no. 2, pp. 191-199, 2016.

[69] S. H. Chan, C. W. Wu, A. F. Li, C. W. Chi, and W. C. Lin, "miR-21 microRNA expression in human gastric carcinomas and its clinical association," Anticancer Research, vol. 28, no. 2A, pp. 907-911, 2008.

[70] L. X. Yan, X. F. Huang, Q. Shao et al., "MicroRNA miR-21 overexpression in human breast cancer is associated with advanced clinical stage, lymph node metastasis and patient poor prognosis," RNA, vol. 14, no. 11, pp. 2348-2360, 2008.

[71] T. Moriyama, K. Ohuchida, K. Mizumoto et al., "MicroRNA-21 modulates biological functions of pancreatic cancer cells including their proliferation, invasion, and chemoresistance," Molecular Cancer Therapeutics, vol. 8, no. 5, pp. 1067-1074, 2009.
[72] Y. Lou, X. Yang, F. Wang, Z. Cui, and Y. Huang, "MicroRNA21 promotes the cell proliferation, invasion and migration abilities in ovarian epithelial carcinomas through inhibiting the expression of PTEN protein," International Journal of Molecular Medicine, vol. 26, no. 6, pp. 819-827, 2010.

[73] X. Xia, B. Yang, X. Zhai et al., "Prognostic role of microRNA21 in colorectal cancer: a meta-analysis," PloS One, vol. 8, no. 11, article e80426, 2013.

[74] L. F. Xu, Z. P. Wu, Y. Chen, Q. S. Zhu, S. Hamidi, and R. Navab, "MicroRNA-21 (miR-21) regulates cellular proliferation, invasion, migration, and apoptosis by targeting PTEN, RECK and Bcl-2 in lung squamous carcinoma, Gejiu City, China," PloS One, vol. 9, no. 8, article e103698, 2014.

[75] M. Dillhoff, J. Liu, W. Frankel, C. Croce, and M. Bloomston, "MicroRNA-21 is overexpressed in pancreatic cancer and a potential predictor of survival," Journal of Gastrointestinal Surgery, vol. 12, no. 12, pp. 2171-2176, 2008.

[76] J. Dong, Y. P. Zhao, L. Zhou, T. P. Zhang, and G. Chen, "Bcl-2 upregulation induced by $\mathrm{miR}-21$ via a direct interaction is associated with apoptosis and chemoresistance in MIA PaCa-2 pancreatic cancer cells," Archives of Medical Research, vol. 42, no. 1, pp. 8-14, 2011.

[77] C. Zhou, J. Ding, and Y. Wu, "Resveratrol induces apoptosis of bladder cancer cells via miR21 regulation of the Akt/Bcl2 signaling pathway," Molecular Medicine Reports, vol. 9, no. 4, pp. 1467-1473, 2014.

[78] R. Q. Miao, J. Agata, L. Chao, and J. Chao, "Kallistatin is a new inhibitor of angiogenesis and tumor growth," Blood, vol. 100, no. 9, pp. 3245-3252, 2002.

[79] L. Lu, Z. Yang, B. Zhu et al., "Kallikrein-binding protein suppresses growth of hepatocellular carcinoma by antiangiogenic activity," Cancer Letters, vol. 257, no. 1, pp. 97106, 2007.

[80] B. Zhu, L. Lu, W. Cai et al., "Kallikrein-binding protein inhibits growth of gastric carcinoma by reducing vascular endothelial growth factor production and angiogenesis," Molecular Cancer Therapeutics, vol. 6, no. 12, Part 1, pp. 3297-3306, 2007.

[81] A. L. Shiau, M. L. Teo, S. Y. Chen et al., "Inhibition of experimental lung metastasis by systemic lentiviral delivery of kallistatin," BMC Cancer, vol. 10, p. 245, 2010.

[82] D. Jia, C. Zheng, J. Feng, J. Zou, and Y. Diao, "Plasmid mediated kallistain gene expression via intramuscular electroporation delivery in vivo for treatment of NCI-H446 subcutaneous xenograft tumor," Pakistan Journal of Pharmaceutical Sciences, vol. 27, 3 Supplement, pp. 633-636, 2014.

[83] Y. Yao, L. Li, X. Huang et al., "SERPINA3K induces apoptosis in human colorectal cancer cells via activating the Fas/FasL/ caspase-8 signaling pathway," The FEBS Journal, vol. 280, no. 14 , pp. 3244-3255, 2013. 


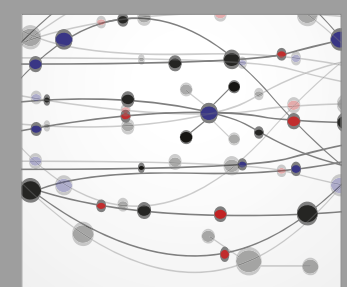

The Scientific World Journal
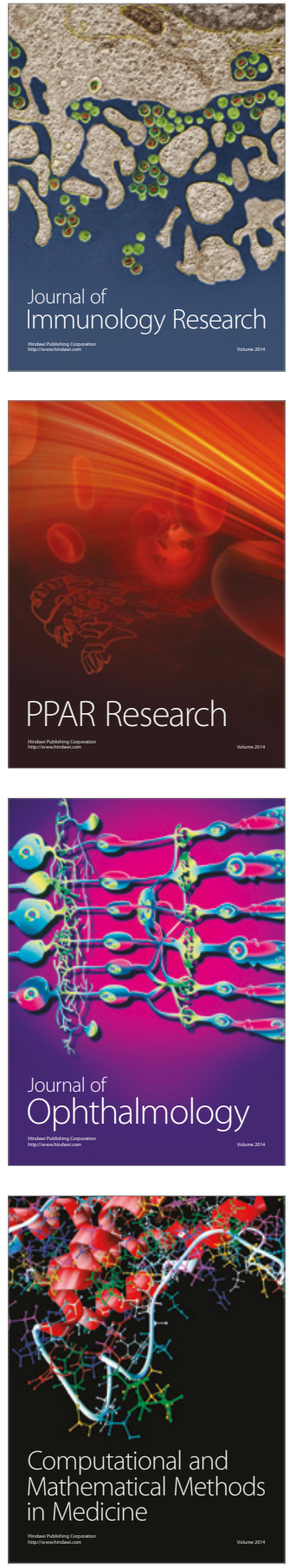

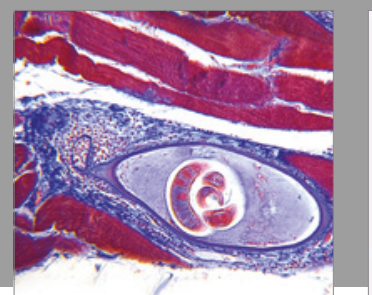

Gastroenterology Research and Practice
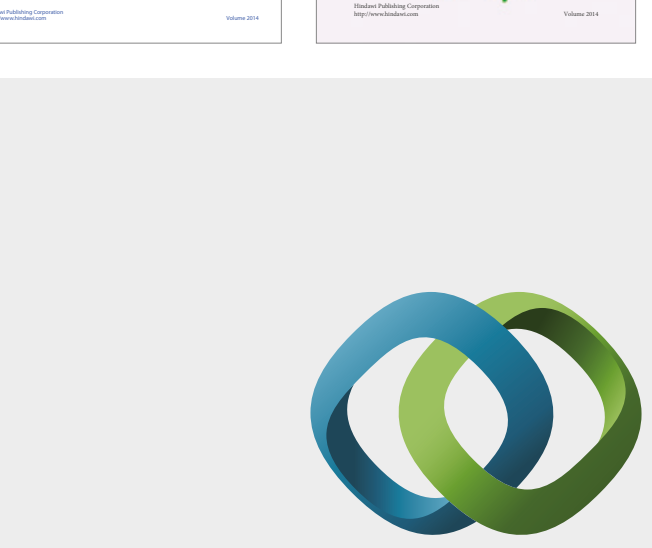

\section{Hindawi}

Submit your manuscripts at

https://www.hindawi.com
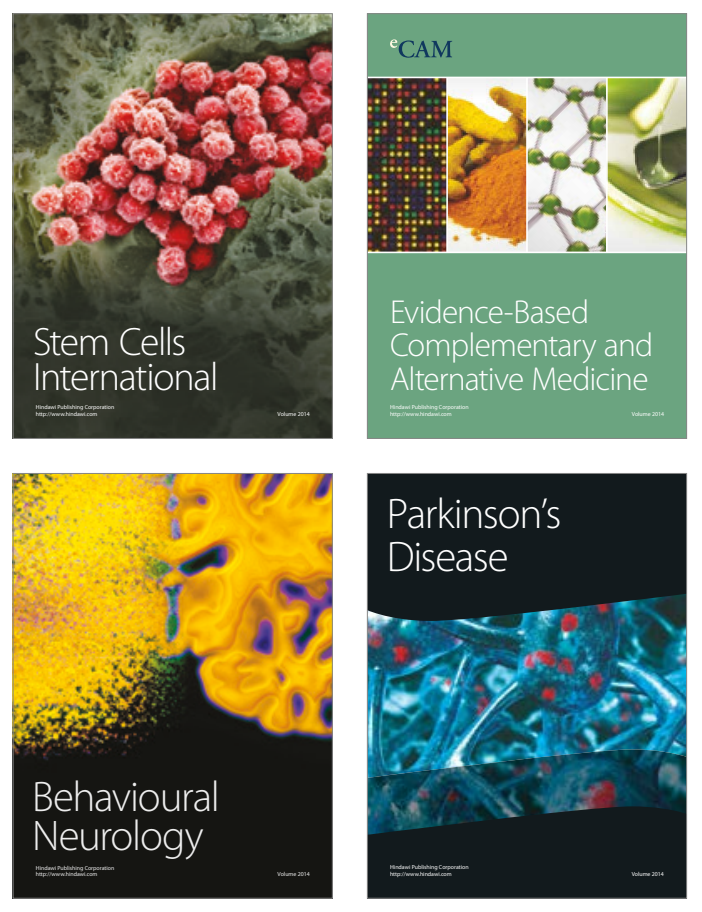
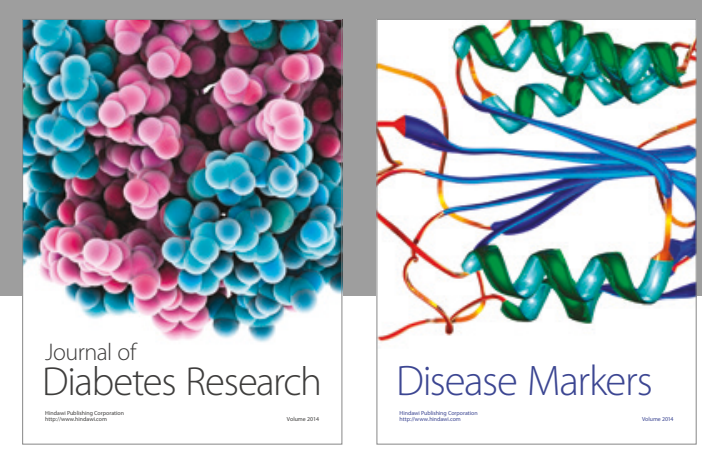

Disease Markers
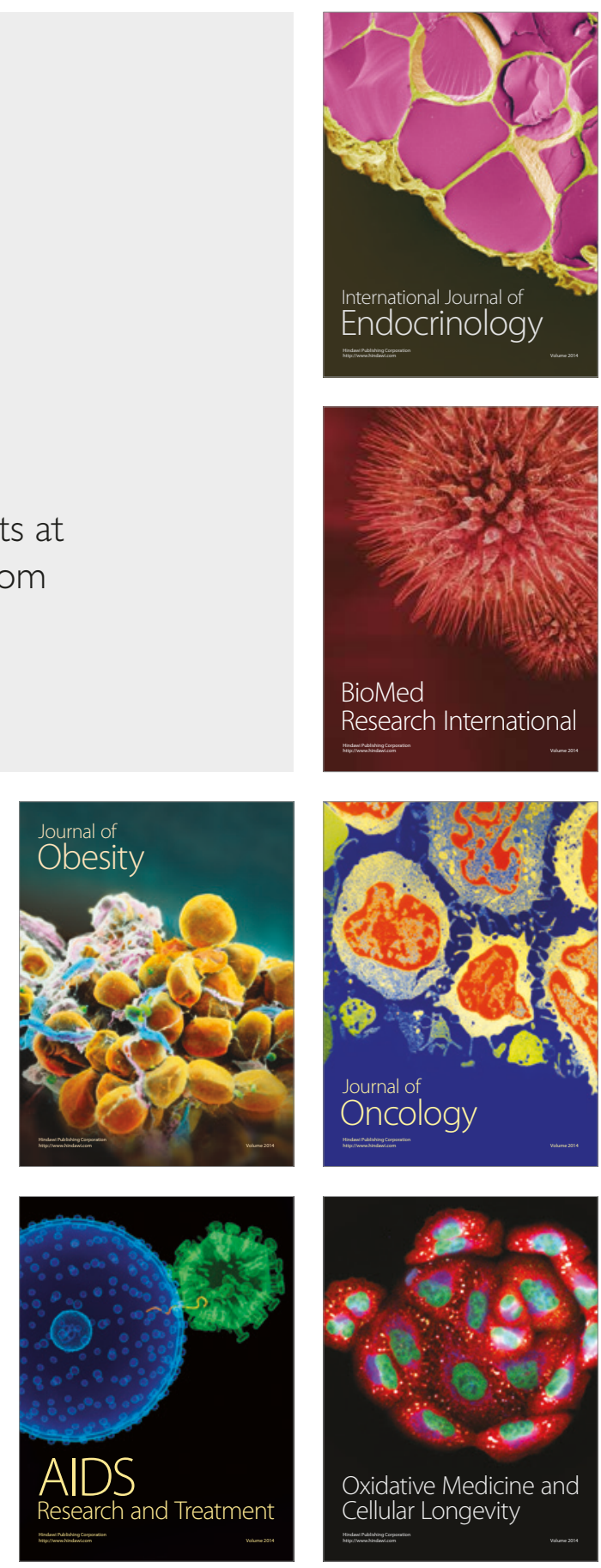